\title{
POLÍTICA DE PRIMERA INFANCIA Y DESARROLLO INFANTIL TEMPRANO EN SERVICIOS NO ESCOLARIZADOS DEL NIVEL INICIAL
}

\section{Early childhood and early childhood development policy in non-service \\ schooled from the initial level \\ https://doi.org/10.47606/ACVEN/PH0086}

\section{Angela Esther Albán Bermejo ${ }^{1^{*}}$ \\ https://orcid.org/0000-0002-6392-322X}

Recibido: 15 julio 2021/ Aprobado: 18 octubre 2021

\section{RESUMEN}

El presente estudio busco reflexionar en torno a la política de primera infancia y el desarrollo infantil temprano en servicios no escolarizados del nivel inicial, dada la importancia que reviste para el desarrollo del país, ya que atender a los niños pequeños de manera pertinente con programas y adultos responsivos favorece poder asegurar su desarrollo integral, sentando las bases socioemocionales, relacionales, cognitivas asegurando el progreso de estos niños, quienes construirán un país más equitativo y con mayores oportunidades. A partir de un enfoque cualitativo que nos llevó a poder reflexionar en torno a las variables del estudio siendo necesario apropiarnos del método inductivo y analítico a través del análisis documental. Las reflexiones finales, invitan a seguir construyendo desde el Estado, una sociedad más justa y horizontal en cuanto a la atención universal de los niños de la primera infancia para garantizar su pleno desarrollo infantil temprano.

Palabras clave: desarrollo infantil temprano, política de primera infancia

1 Universidad César Vallejo. Piura, Perú.

* Autor de correspondencia: angelitaab.ab@gmail.com 


\begin{abstract}
The present study seeks to reflect on early childhood policy and the early childhood development in non-school services of the initial level, given the importance for the development of the country, since caring for children young children in a relevant way with responsive programs and adults favors be able to ensure their integral development, laying the socio-emotional foundations, relational, cognitive, ensuring the progress of these children, who they will build a more equitable country with greater opportunities. From a qualitative approach that led us to reflect on the variables of the study being necessary to appropriate the inductive and analytical method through the documentary analysis. The final reflections invite you to continue building from the State, a more just and horizontal society in terms of universal care of early childhood children to ensure their full child development early.
\end{abstract}

Keywords: early childhood development, early childhood policy

\title{
INTRODUCCIÓN
}

En los últimos años a nivel mundial, encontramos que los Objetivos de Desarrollo Sostenible (ODS) proponen oportunidades históricas para la implementación a gran escala de intervenciones que favorecen desarrollo infantil temprano (DIT), siendo que la evidencia ha aumentado sobre todo en sectores, poblaciones y entornos vulnerables encontrando que las deficiencias físicas y nutricionales traen consecuencias de mayor alcance para la vida de esta población infantil (Britto et al., 2016). En ese marco el gobierno chino, asumió desde el año 2012 que la calidad de la educación de un país es un indicador de las perspectivas generales de desarrollo de un país (Greubel y Van-Der, 2012).

Pérez-Escamilla et al., (2017) también coinciden en afirmar que el DIT es la base del progreso de un estado tanto en lo económico como en lo social y su capacidad para conseguir los ODS penderán de esa voluntad que puedan ejercer sus gobernantes para contrarrestar el retraso del crecimiento físico que resulta la consecuencia de mayor trascendencia para la salud del infante y que repercute en su desarrollo mental e intelectual, de igual forma Verch (2017), agrega que tanto el bienestar social así como la atención durante el periodo que va hasta los cinco años del niño son importantes como base para el desarrollo de los aspectos biopsicosociales y también cognitivos relacionados con su salud, con el 
aprendizaje, con la educación y con el entorno familiar favoreciendo las capacidades de aprendizaje tanto en el aula como en la comunidad.

Sin embargo, en este contexto los administradores públicos y los formuladores de políticas y programas de desarrollo infantil temprano, demuestran poca efectividad, obteniendo resultados poco favorables en términos económicos, no dando lugar a importantes iniciativas nacionales y mundiales (Castro et al., 2021). En América Latina y el Caribe, después de estudios longitudinales en Guatemala, Brasil y Jamaica, llegaron a la conclusión de que el cuidado de la primera infancia como base para nuevos aprendizajes sí tiene un efecto beneficioso sobre el desarrollo de la primera infancia (Grantham y Rubio, 2020). Asimismo, los lactantes que no reciben la atención adecuada presentan discapacidades cognitivas, socioemocionales y físicas, muestran un bajo rendimiento escolar en los años siguientes y también reciben menores ingresos en la edad adulta (Vásquez, 2020). En nuestro país, la evidencia científica internacional ha llevado a la propuesta de una política de primera infancia denominada Estrategia de Gestión Territorial, Primero la Infancia, cuyos resultados son "partos sanos, apego de tipo seguro, una adecuada nutrición, una efectiva comunicación verbal, un desarrollo motor autónomo para caminar, autorregulación de las emociones, así como del comportamiento, y la función simbólica" (MIDIS, 2019a, s/p).

Los resultados mostrados por el ENDES sobre esta política de primera infancia representan un avance significativo en la comprensión del progreso del desarrollo infantil temprano y en el establecimiento de la lógica general de intervención. En cuanto a los resultados, nos muestra desventajosos porcentajes como lo es por ejemplo que el $52,2 \%$ de infantes de nueve meses a treinta y seis meses no lograron una efectiva comunicación verbal a nivel integral y también expresivo. De igual forma estas desventajas se evidencian en los demás indicadores de la estrategia (INEI, 2020), lo que nos lleva a preguntarnos ¿cómo es que se alcanzan estos porcentajes tan desfavorables para los niños pequeños, más aún si se suma 
a ello las estadísticas alarmantes de anemia y desnutrición crónica que perjudica de manera directa al desarrollo infantil temprano de nuestros niños (MIDIS, 2019b).

Por ello resulta necesario señalar el estudio realizado por Guerrero (2019) quien nos habla sobre el trabajo que se viene ejecutando para garantizar este desarrollo, por ello la medición de estos avalaría que se pueda medir y a su vez revelar tanto avances como desafíos de políticas de primera infancia en el territorio.

El sector educativo, a través de los "Programas de Educación Inicial No Escolarizados" (PRONOEI), reciben a los niños del grupo correspondiente de cero a treinta y seis meses. Estos escolares son acompañados por el personal al que se le designa como "Promotores Educativos Comunitarios" (PEC), siendo importante mencionar que si bien se cuenta con la política de primera infancia, el buen desarrollo infantil temprano sigue en riesgo, ello se refleja por ejemplo en que 1 de cada 5 niños en zonas rurales sufre de desnutrición de tipo crónica (SIREPI, 2020) por otro lado se resaltan las grandes falencias en la atención de estos niños al ser comparados con la franja etaria que nos muestra los comportamientos propios de los niños según las edades alcanzadas. En este marco el presente artículo se planteó como objetivo reflexionar en torno a la política de primera infancia y el desarrollo infantil temprano en servicios no escolarizados del nivel inicial.

\section{METODOLOGÍA}

El estudio se construyó bajo la orientación del enfoque cualitativo con el objetivo de reflexionar en torno a la política de primera infancia y el desarrollo infantil temprano en servicios no escolarizados del nivel inicial. Al respecto Sánchez-Flores (2019) nos dice que el enfoque cualitativo

se sustenta en evidencias que se orientan más hacia la descripción profunda del fenómeno con la finalidad de comprenderlo y explicarlo a través de la aplicación de métodos y técnicas derivadas de sus concepciones y fundamentos epistémicos, como la hermenéutica, la fenomenología y el método inductivo $(\mathrm{s} / \mathrm{n})$. 
El estudio asumió el método inductivo, en palabras de Rodríguez-Jiménez y PérezJacinto (2017) la inducción es una forma de razonamiento en la que las personas pasan del conocimiento de casos específicos a un conocimiento más general, que refleja el terreno común en los fenómenos individuales. Se basa en repetir hechos y fenómenos en la realidad, encontrar características comunes en un grupo definido y sacar conclusiones sobre los aspectos que lo caracterizan y donde la generalización extraída se basa en la experiencia; de esta manera este método ayudo a poder construir el conocimiento en torno a las variables política de primera infancia y el desarrollo infantil temprano.

El método analítico complementó el estudio en la medida que contribuyó en la búsqueda de la información. Al respecto Rodríguez-Jiménez y Pérez-Jacinto ob cit., mencionan que el análisis es un proceso lógico, el cual puede descomponerse mentalmente, es decir un todo en sus múltiples partes y cualidades, en sus múltiples relaciones, atributos y elemento; de esta manera este método permitió buscar la información a través de acciones que conllevaron a un pensamiento lógico sobre el comportamiento de cada una de las variables política de primera infancia y el desarrollo infantil temprano.

\section{Recolección de la información}

Para la recolección de información se utilizó el análisis documental como técnica para obtener una serie de datos como referencia para formar los aspectos teóricos que sustentaron la investigación. Este análisis es una técnica para estudiar y analizar información de manera sistemática, cuantitativa y objetiva, que permite inferencias a partir de datos e información confiables y válidos basados en el contexto (Cadena-Iñíguez et al., 2017).

\section{Procesamiento de la información}

Para procesar la información se utilizó archivos de texto, archivos de resúmenes y archivos de paráfrasis de artículos científicos, artículos, revistas y libros, que 
ayudaron a organizar y presentar las teorías e investigaciones esperadas en esta investigación. Ríos (2017) nos dice que son "herramientas para el registro de datos o información de fuentes bibliográficas" (p. 105).

\section{Análisis de la información}

El análisis descriptivo tiene como objetivo proporcionar una visión general y respuestas objetivas a preguntas que surgen en una determinada parte de la realidad y el conocimiento, estableciendo teorías científicas que se puedan utilizar con fines de investigación. Al respecto, Ríos (2017) nos dice que el análisis descriptivo "es el segundo nivel de conocimiento, que busca descubrir las características, comportamiento y atributos del objeto de investigación" (p. 81).

\section{RESULTADOS Y DISCUSIÓN}

\section{Visión global de la primera infancia}

Ebrahim, Okwany \& Barry (2018) defienden que

La importancia del cuidado y la educación de la primera infancia (AEPI) en la vida de los niños muy pequeños está ganando cada vez más atención en todo el mundo y, sin embargo, existe una falta persistente de diversas perspectivas de conocimiento sobre esta fase crítica (p.12).

Por lo tanto, los adultos como los niños están sujetos a vulnerabilidades superpuestas que son contraproducentes para su bienestar. Estos niños seguirán sufriendo exclusión y desigualdad si los hogares pobres no reciben el apoyo adecuado. Sostiene que el sistema educativo no ha logrado proteger el derecho de los niños pequeños al cuidado y la educación al no universalizar la cobertura, dejando la provisión al sector privado.

Por su parte Bipath \& Theron (2020) considera que:

La educación de la primera infancia puede tener un impacto positivo en la vida, el bienestar, la seguridad, el crecimiento, el desarrollo y el rendimiento académico de los niños pequeños en el grupo de edad desde el nacimiento hasta los 4 años (p. 227). 
En el contexto actual del desarrollo de la primera infancia asociada a la escolarización los autores ponen énfasis en la adquisición de habilidades pre académicas específicas y la transferencia de conocimientos por parte del adulto, en lugar de un enfoque en objetivos amplios de desarrollo como el bienestar socioemocional y la adquisición de comprensión y conocimiento por parte del niño a través de la experiencia directa y la experimentación.

Por su parte Ring \& O'Sullivan (2018) presentan una visión más sensible al resaltar que "La educación de la primera infancia de alta calidad se asocia con la provisión de un plan de estudios centrado en el niño basado en el conocimiento de cómo aprenden los niños pequeños" (p. 229). En ese sentido, La atención de los niños pequeños se encuentra actualmente en un período decisivo, caracterizado por una situación incierta en el sistema educativo. La educación social se centra en el desarrollo de las habilidades sociales, los juegos y la interacción de los niños, combinando educación y cuidado, y teniendo una visión integral del desarrollo infantil.

Por tanto, resulta importante mencionar a UNICEF (2017) cuando refiere que "la primera infancia es igualmente un período crítico para afianzar los beneficios de las intervenciones tempranas y reducir los efectos negativos de los riesgos" (p.2). Ello implica que el desarrollo de los niños en la primera infancia se fortalece a través de programas de apoyo para sus padres, la provisión de estrategias y técnicas de crianza y el apoyo financiero para los padres que ayudan a reducir los riesgos de maltrato físico en niños; la participación de un adulto en el cuidado de los niños puede contribuir a una variedad de resultados positivos para las niñas, los niños y la familia en su conjunto, como la reducción de la violencia.

Por su parte Grantham-McGregor y Rubio-Codina (2020), mencionan que

Existe un creciente compromiso a nivel mundial para llevar a cabo intervenciones en la primera infancia con el objetivo de promover el desarrollo de millones de niños en situación de desventaja en países 
de ingresos bajos y medios que no logran alcanzar su pleno potencial de desarrollo. No obstante, los progresos en esta área se ven obstaculizados por la falta de pruebas de desarrollo factibles de ser usadas a escala. Es por esto por lo que contar con este tipo de pruebas es una necesidad de carácter urgente $(\mathrm{s} / \mathrm{n})$.

Es importante entonces reconocer que la base de la salud y el bienestar de los adultos se sientan temprano en la vida, el mundo está cada vez más comprometido con la implementación de intervenciones para promover la escala del desarrollo de la primera infancia (DIT), con el objetivo de resolver este problema y promover el desarrollo de los niños. Sin embargo, el progreso de estos esfuerzos se ve obstaculizado por la falta de herramientas de medición del desarrollo infantil confiables y efectivas que puedan recopilar datos fácilmente, especialmente para los niños menores de 3 años. Estas herramientas son esenciales para monitorear y evaluar la efectividad de las intervenciones diseñadas e implementadas y para medir los niveles de DIT a nivel poblacional.

\section{La política de primera infancia}

Es importante mencionara a Verch ob cit., cuando resalta que las diferencias entre los niños se evidencian desde la infancia. En ese contexto, se han desarrollado políticas públicas en todo el mundo, políticas revolucionarias destinadas a promover el desarrollo infantil de todas las familias de la mejor manera posible. De esta forma, los cuidadores, ya sean padres, familiares o incluso especialistas sin vínculos familiares, ejercen una influencia decisiva y transformadora y ayudan a moldear la mente y el cerebro del niño, lo que repercutirá durante toda la vida.

Por su parte Grantham-McGregor y Rubio-Codina ob cit., mencionan que

Existe un creciente compromiso a nivel mundial para llevar a cabo intervenciones en la primera infancia con el objetivo de promover el desarrollo de millones de niños en situación de desventaja en países de ingresos bajos y medios que no logran alcanzar su pleno potencial de desarrollo. No obstante, los progresos en esta área se ven obstaculizados por la falta de pruebas de desarrollo factibles de ser usadas a escala $(\mathrm{s} / \mathrm{p})$. 
Es importante entonces reconocer que la base de la salud y el bienestar de los adultos se sientan temprano en la vida, el mundo está cada vez más comprometido con la implementación de intervenciones para promover la escala del desarrollo de la primera infancia (DIT), con el objetivo de resolver este problema y promover el desarrollo de los niños. Sin embargo, el progreso de estos esfuerzos se ve obstaculizado por la falta de herramientas de medición del desarrollo infantil confiables y efectivas que puedan recopilar datos fácilmente, especialmente para los niños menores de 3 años. Estas herramientas son esenciales para monitorear y evaluar la efectividad de las intervenciones diseñadas e implementadas y para medir los niveles de DIT a nivel poblacional, y aquí nos preguntas ¿qué se viene haciendo para obtener instrumentos validados propios de un país que respondan a las características de su población infantil y que permitan recoger los indicadores del DIT.

EI MIDIS (2019a) describe que la "La política de primera infancia es un hito en las decisiones tomadas por el gobierno peruano, que permite construir consensos pertinentes enmarcados en un conjunto de acciones estratégicas que conducen hacia el qué hacer para solidificar de manera articulada resultados para mejorar el desarrollo de los infantes del Perú" (p.35).

Las mejoras de las condiciones de los niños en Perú son una prioridad para el gobierno, que reconoce que los niños son las manifestaciones más visibles y expresivas del desarrollo humano y social de un país. Por tanto, la política peruana sobre la primera infancia es un momento de referencia porque, antes de acordar las acciones, se ha establecido una dirección estratégica con una idea clara de lo que hay que hacer para avanzar con acciones coordinadas hacia futuro (MIDIS, 2019a, p. 35).

Y si bien los resultados mostrados por ENDES sobre esta política de primera infancia representan un gran avance en la comprensión del desarrollo de la 
primera infancia y en el establecimiento de la lógica general de intervención. Surge una contradicción en cuanto a los resultados, que nos muestra un porcentaje desfavorable, por ejemplo, el $52,2 \%$ de los pequeños entre 9 y 36 meses no lograron una comunicación oral efectiva a nivel general y expresivo. De igual manera, estas desventajas también son evidentes en otros indicadores de la estrategia (INEI ob cit.), lo que hace que nos preguntemos cómo, una política que invierte millones de soles para su implementación conlleve a obtener estos resultados de gran desventaja en los niños pequeños a quienes también se les suma los altos índices de anemia y desnutrición crónica que perjudica directamente el desarrollo de los niños de la primera infancia de nuestro país (MIDIS, 2019b).

Por su parte el Sistema regional de Primera Infancia (SIREPI) de la Región Piura, implementa también esta política de primera infancia y si bien desde el año 2013 fecha en que creo este sistema ha venido trabajando por la primera infancia digamos que ha logrado algunos beneficios como el hecho de instalar comités distritales en la mayoría de las provincias de la región, contar con un PIP que les ha permitido implementar el programa "Familia Feliz", sin embargo, encontramos que el índice de anemia y desnutrición en los niños piuranos contempla que 1 de cada 5 niños en zonas rurales sufra de desnutrición de tipo crónica (SIREPI, 2020).

\section{Desarrollo infantil temprano y los servicios de primera infancia}

Castro et al., (2020) al mencionar que "El desarrollo infantil temprano (DIT) es el proceso de madurez de habilidades, movimiento, cognición, lenguaje, emoción social y habilidades de adaptación hasta los treinta y seis meses de vida, lo que conlleva al desarrollo de los aspectos cognitivos, físicos, sociales y emocionales" (p. 533).

Se define entonces como la madurez gradual de las habilidades, lo motriz, la cognición, el lenguaje y las emociones sociales de los infantes en los primeros sesenta meses de vida. El desarrollo infantil es un proceso en el que las 
habilidades y capacidades de adaptación antes mencionadas maduran gradualmente durante los primeros cinco años de vida. EI DIT se basa en un conjunto de dimensiones que contempla lo físico, lo emocional, lo social y lo cognitivo y que deben ser consideradas de manera holística (Castro et al. ob cit., p. 35).

Para Díaz et al., (2017) "El desarrollo infantil, caracterizado por la progresiva adquisición de estructuras y funciones durante las etapas tempranas de la vida, está influido por las condiciones socioeconómicas de los niños y sus familias" $(p, 1)$.

Entonces, las intervenciones destinadas a promover el desarrollo de la primera infancia enfrentan dificultades para establecer líneas de base y evaluar su impacto, porque las escalas que se pueden utilizar para evaluar el desarrollo infantil son ineficaces en cualquier contexto social y cultural, y no pueden Aplicado a nivel poblacional. Por lo tanto, las condiciones que existen durante el período prenatal y los primeros años de vida explican la mayoría de los cambios de salud.

Gallardo (2017) resalta que:

Esta etapa de Educación Infantil tiene una gran importancia en la posterior evolución de la persona. En estos primeros años se configuran las estructuras neuronales y se produce un crecimiento físico, psicomotor, perceptivo, intelectual tan rápido como no va a suceder en ninguna de las etapas posteriores, y se van a realizar los procesos de individualización y socialización (p. 6).

La edad inicial constituye un período importante, que se caracteriza por un ritmo de evolución y desarrollo fuerte y en constante cambio, durante el cual la plasticidad y flexibilidad de la estructura física y psicológica de los niños y niñas adquieren y poseen el mayor índice decisivo para su posterior desarrollo. Desde esta perspectiva, cualquier desviación de capacidad debe ser compensada lo antes posible para evitar restricciones a su evolución posterior. En la actualidad, departamentos de administración educativa, docentes y familias coinciden en que 
la etapa de educación infantil es un aporte decisivo al desarrollo y aprendizaje de los niños desde los primeros años de vida

EI MIDIS (2016) plantea que el DIT:

Es un proceso progresivo, multidimensional, integral y oportuno que se traduce en la construcción de capacidades cada vez más complejas, que permiten a la niña y niño ser competentes a partir de sus potencialidades para lograr una mayor autonomía en interacción con su entorno en pleno ejercicio de sus derechos (p. 9).

En ese sentido las niñas y los niños se constituyen en sujetos de derechos por su condición de seres humanos. Por lo que se les considera:

(i) Personas sociales, porque son parte de la familia desde el nacimiento, y la familia está inmersa en el trasfondo social y cultural;

(ii) Existencia única, porque pasan por un proceso de personalización, en el que van construyendo y desarrollando capacidades y potencialidades;

(iii) La existencia de diversidad, porque se han desarrollado en un mundo diverso desde su nacimiento, ya sea desde una perspectiva cultural, personal (género y edad), o desde una perspectiva social.

Por tanto, el Estado y la sociedad deben reconocer y garantizar los derechos de las niñas y los niños, y darles un estatus por encima de otros intereses y consideraciones

Sin embargo, cuando mencionamos los servicios no escolarizados Betanzos-Lara y Villaseór-Palma (2019) nos dice que los infantes en edad escolar temprana pueden estar excluidos por causas relacionadas al contexto donde están inmersos.

Esta conclusión dada por la autora revela una dura realidad ya que estos servicios de ciclo I a cargo del MINEDU no cuentan con los recursos necesarios a diferencia del programa Cuna Más a cargo del MIDIS quien a su vez se encarga de la política de primera infancia en el Perú. 
Vemos entonces como la política si bien esta dada ya, esta no es implementada de manera universal, si no que crea brechas y desigualdad entre los niños de 0 a 3 años del país. Estos hallazgos, se corroboran cuando Gutiérrez (2021) muestra los resultados de su investigación concluyendo que en relación al nivel de conocimiento del desarrollo infantil temprano en las PEC, un $46.7 \%$ de ellas, se ubicó en nivel bajo. Lo que indica que las promotoras desconocen sobre el proceso de crecimiento, maduración y desarrollo de los niños menores de 3 años.

Ello demuestra, las limitaciones en las que vienen siendo atendidos los niños menores de 3 años de los Programas No escolarizados de Educación Inicial de la Región Piura, recordemos que esta etapa tan sensible y trascendental en la vida del niño requiere de adultos responsivos para atender sus necesidades y también brindar condiciones que favorezcan y aseguren su desarrollo integral.

Por otro lado, una pieza fundamental en este acompañar el desarrollo de los niños pequeños es sin dudad los padres, quienes juegan un papel importante en el desarrollo de sus hijos, su estilo de crianza puede ser una herramienta para compensar la desigualdad que brinda el contexto (Betanzo-Lara y Villaseór-Palma ob cit.). Los resultados de la investigación nos permiten comprender cómo los padres que participan en el programa integran las prácticas de crianza y los métodos inclusivos en el cuidado y la educación de sus hijos.

La inclusión de estas prácticas incide principalmente en las áreas socioemocionales y cognitivas del desarrollo de los niños pequeños, lo que les ayuda a avanzar en ciertos indicadores de desempeño de los estándares curriculares. Nuestra conclusión es que al adoptar un enfoque inclusivo de las prácticas parentales, es posible apoyar la inclusión de niños pertenecientes a ciertos entornos frágiles en las escuelas formales y reducir el rezago en su desarrollo y desempeño curricular, lo que afectará sus posibilidades de aprendizaje. Aprendizaje a lo largo de la vida y participa. 
Asimismo, cuando se habla del desarrollo emocional en la primera infancia Guil et al. (2018) mencionan que "El desarrollo emocional del infante y el papel de las emociones en el establecimiento de relaciones sociales, es la base para abordar los procesos de socialización y el papel de las emociones en el desarrollo infantil" ( $p$. 4). Es decir antes del desarrollo del lenguaje, la emoción constituyó el primer sistema de comunicación humano. La interacción social más básica y una de las más importantes se obtiene a una edad muy temprana. Se define como la capacidad de percibir y reconocer las propias emociones. Implica la decodificación precisa de señales emocionales en expresiones faciales, entonación o expresiones corporales. Según Wallon, además de su valor adaptativo, las emociones también se ven afectadas por factores genéticos, porque produce nuevas estructuras cognitivas que brindan a los individuos la existencia clave de los signos de identidad, emoción y lenguaje. La base de su personalidad es biológica, su composición y establecimiento están más controlados por el intercambio social. Debido a y a través de las emociones, los bebés se transforman de una existencia biológica a una existencia social. Muestra que el estado emocional global se relaciona gradualmente con eventos en el entorno de los niños a través del proceso de condicionamiento clásico y operacional. Son estos los que actúan como vínculo entre el proceso puramente orgánico y el medio ambiente; lo mismo ocurre entre el cuerpo y la mente.

Otro aspecto del desarrollo infantil temprano está relacionado al vínculo de apego por ello Kamza (2019) añade que "Según la teoría del apego los sistemas de exploración y fijación son inextricablemente vinculado: los niños exploran su entorno cuando se sienten protegidos y confortados por su cuidador" (p.7). El sistema de apego se activa principalmente por la amenaza psíquica y sirve para proteger al bebé. Actualmente, se afirma que la relación de apego es más bien específico del día; Por lo tanto, el apego con la madre puede ser diferente a la del padre u otro cuidador 
Por su parte el PCEI (2017) menciona que "El desarrollo personal y social de nuestros niños y niñas es un proceso que se inicia en la familia y se construye sobre la base de las relaciones seguras y afectivas que establecen con las personas que los cuidan" (p. 70).

Entonces estas relaciones significativas forman el vínculo de apego, proporcionándoles la necesaria sensación de seguridad y tolerancia, permitiéndoles convertirse en personas únicas y especiales e interactuar con el mundo. Este vínculo también les permite confiar en los adultos que los cuidan y acompañan. En los primeros meses de vida, el niño siente que él y su madre son uno. A partir de la interacción respetuosa y afectuosa con ella, a medida que va creciendo, puede distinguir su cuerpo del de su madre, sintiéndose una persona diferente. Este proceso de separación facilita su transición a una sociedad llena de confianza y seguridad, y puede explorar y mostrar interés hacia el mundo.

EI PCEI ob cit., nos dice también que "En el proceso de su desarrollo psicomotriz, los niños y las niñas viven su cuerpo a través de la libre exploración y experimentación de sus movimientos, posturas, desplazamientos y juegos, en interacción permanente con su entorno y ambiente" (p. 18).

Por tanto durante los primeros meses de vida, el cuerpo y el movimiento son los principales medios que utilizan los niños y niñas para expresar sus deseos, sentimientos y emociones, para ir en busca del mundo que está ahí para ser explorado y conquistado. De esta forma, el bebé adquiere gradualmente la primera postura —por ejemplo, de boca arriba a boca abajo, y viceversa, sentado, arrodillado y de pie - hasta que alcanza la verticalidad y continúa ampliando sus posibilidades de movimiento y acción. Estas experiencias les han dado un conocimiento más profundo de su cuerpo y las posibilidades de sus acciones y expresiones, han aprendido a tener un mayor dominio, control y coordinación de sus cuerpos, movimientos y habilidades motoras, beneficiándolos así en la construcción de su propia imagen corporal. 
De igual forma el PCEI ob cit., resalta que:

En las interacciones con el entorno descubren el placer de comunicarse y transitan de la comunicación no verbal a la comunicación verbal. Es así como, a través de los gestos, miradas, movimientos corporales y primeros balbuceos, expresan a otros sus necesidades e intereses, emergiendo sus primeras palabras como producto de estas relaciones y vivencias (p.16).

La comunicación es fundamental y absolutamente necesaria, Esto surge desde el comienzo de la vida, acompañado por la alegría de la interacción y la transformación mutua. Por lo tanto, en los primeros años de vida, es importante tratar a los bebés o niños como interlocutores efectivos con la capacidad de comunicación y expresión. En este tiempo de interacción con los adultos, los niños y niñas se comunicaban a través de balbuceos, sonrisas, expresiones faciales, llantos y gestos para expresar sus necesidades, emociones, intereses o experiencias.

Asimismo, el PCEl ob cit., hace hincapié que:

Los niños y niñas, desde que nacen, son sujetos de acción, e interactúan con las personas, los objetos y seres vivos que forman parte de su ambiente. Desde sus posibilidades de movimiento, desplazamiento y emoción en su cotidianidad, empiezan a explorar espontáneamente el entorno que los rodea y a vivir experiencias que les permiten poco a poco ir descubriendo el mundo (p. 149).

En ese sentido, en el área descubrimiento del mundo el niño demuestra su curiosidad natural y la seguridad emocional dada por un adulto sensible los alienta a explorar y experimentar con todo su cuerpo por sí mismos, y estar atentos a los eventos que les suceden y las situaciones que suceden a su alrededor. Al chupar, morder, mirar, oír, oler, tocar, cargar, golpear, tirar, rotar, experimentan y comienzan a descubrir algunos rasgos perceptivos para asignar usos o reconocer objetos en su entorno; Empiezas a compararlos, ordenar, compilar y agrupar según sus propios criterios. Los niños y las niñas se vuelven gradualmente más complejos en sus acciones sobre los objetos y con un mayor dominio de sus 
posturas y movimientos, se mueven y descubren algunas relaciones espaciales entre su cuerpo y las personas y objetos en su entorno. A partir de estas acciones exploratorias construyen sus primeros conceptos de espacio, cantidad, tiempo y causalidad, así como sus ideas. qué es y qué está sucediendo en el mundo. En estas acciones, los niños cambian y transforman su entorno; Encuentra significados que le dan sentido a esa experiencia realizada y así amplía sus formas de explorar y de conocer el mundo.

\section{CONCLUSIONES}

a) La política estrategia "Primero la infancia" su implementación no ha obtenido los resultados esperados, lo que implica la falta de compromiso social y voluntad política para garantizar un óptimo y pertinente desarrollo infantil temprano (DIT) en el grupo de edad de 0 a 3 años

b) Que los Servicios no escolarizados de educación inicial de la Región Piura no brindan una atención pertinente al encontrar que el adulto encargado de acompañar el proceso de desarrollo de los niños, en su mayoría no cuenta con los conocimientos mínimos sobre DIT

\section{RECOMENDACIONES}

a) Instalar equipos multisectoriales organizados para afrontar las necesidades urgentes en la atención de los niños menores de 3 años

b) Como política urgente de gestión, se debe tomar acciones efectivas para enfrentar el impacto del trabajo en la región Piura que atiende a niños del grupo etario de 0 a 2 años en los PRONOEI del ciclo I. Se tiene como actores en cada una de las UGEL al Área de Educación Inicial y los Servicios no Escolarizados de educación Inicial para la implementación de estrategias que conlleven a tener servicios pertinentes y oportunos para este grupo de edad. 


\section{REFERENCIAS}

Betanzos Lara NA y Villaseór Palma KM (2019). Programas de atención y educación de la primera infancia en exclusión de América Latina. Revista de Educación y Desarrollo, 5. Recuperado de: https://www.cucs.udg.mx/revistas/edu desarrollo/anteriores/51/51 Betanzos.pdf

Bipath, K., \& Theron, H. (2020). Contesting schoolification through snapshots of pedagogy-in-participation in early childhood development centres in South Africa. Perspectives in Education, 38(2), 227-240. https://doi.org/10.18820/2519593X/pie.v38.i2.15

Britto, P., Lye. S, Proulx, K., Yousafzai, A. (2016). Nurturing care: promoting early childhood development. Lancet. Recuperado de: https://www.thelancet.com/iournals/lancet/article/PIIS0140-6736(16)31390-3/fulltext

Cadena-Iñíguez P, Rendón Medel R, Aguilar Ávila J, Salinas Cruz E, de la CruzMorales F del R y Sangerman Jarquin DM. (2017). Métodos cuantitativos, métodos cualitativos o su combinación en la investigación: un acercamiento en las ciencias sociales. Instituto Nacional de Investigaciones Forestales, Agrícolas y Pecuarias. Recuperado de: https://www.redalyc.org/journal/2631/263153520009/html/

Castro, F., Vázquez, R., Villalobos, A., Rubio, M., Prado, E., Sánchez, J., Romero, M. y Shamah, T. (2021). Contexto y resultados del desarrollo infantil temprano en niños y niñas de 12 a 59 meses en México. Recuperado de: https://www.scielosp.org/article/spm/2019.v61n6/775-786/es/

Castro, F., Rojas-Martínez, R., Villalobos, A., Shamah, T., Vázquez-Salas, R. A., Armendares, N., Allen-Leigh, B., Escamilla, A., \& Romero, M. (2020). Bases metodológicas de la medición de desarrollo infantil temprano en la Ensanut 100k. Salud Pública De México, 62(5), 532-539. Recuperado de: https://www.saludpublica.mx/index.php/spm/article/view/11067

Díaz, A. A., Gallestey, J. B., Vargas-Machuca, R., \& Velarde, R. A. (2017). Desarrollo infantil en zonas pobres de Perú [Child development in poor areas of Peru]. Revista panamericana de salud pública $=$ Pan American journal of public health, 41, e71. https://doi.org/10.26633/RPSP.2017.71

Ebrahim, H.B., Okwany, A., \& Barry, O. (Eds.). (2018). Early Childhood Care and Education at the Margins: African Perspectives on Birth to Three (1st ed.). Routledge. https://doi.org/10.4324/9781351185158

Gallardo, E. G. (2017). La atención temprana como recurso para la mejora del proceso de nueva escolarización en el segundo ciclo de la etapa de educación infantil, del alumnado con trastornos en el desarrollo o en riesgo de padecerlo, en Huelva y su provincia [Http://purl.org/dc/dcmitype/Text, Universidad de Huelva]. Recuperado de: https://dialnet.unirioja.es/servlet/tesis?codigo=136777

Grantham McGregor, S. y Rubio Codina, M. (2020). Validez predictiva de pruebas cortas comúnmente usadas para medir el desarrollo infantil en estudios a gran 
escala. Banco Interamericano de Desarrollo. Recuperado de: https://publications.iadb.org/publications/spanish/document/Validez-predictiva-de-pruebascortas-comunmente-usadas-para-medir-el-desarrollo-infantil-en-estudios-a-gran-escala.pdf

Greubel, L. y Van Der, J. (2012). Early Childhood Development: A Chinese National Priority and Global Concern for 2015. Recuperado de: https://www.thelancet.com/iournals/lancet/article/PIIS0140-6736(16)31390-3/fulltext

Guerrero, G (2019). Perú: Informe de Progreso de Políticas de Primera Infancia. Lima: Diálogo Interamericano; GRADE. Recuperado de: http://www.grade.org.pe/publicaciones/peru-informe-de-progreso-de-politicas-de-primerainfancia/

Guil, R., Mestre, J. M., Gil Olarte, P., De la Torre, G. G., y Zayas, A. (2018). Desarrollo de la inteligencia emocional en la primera infancia: una guía para la intervención. Universitas Psychologica, 17(4), 1-12. https://doi.org/10.11144/Ja veriana. upsy17-4.diep

Gutierréz, I. (2021). Propuesta DAIS para mejorar el conocimiento del desarrollo infantil temprano en las promotoras educativas comunitarias de UGEL Piura $2020 . \quad$ Recuperado de: https://repositorio.ucv.edu.pe/bitstream/handle/20.500.12692/61761/Gutierrez RIDSSD.pdf? sequence $=1 \&$ isAllowed $=\mathrm{y}$

INEI. (2020). Desarrollo Infantil Temprano en niñas y niños menores de 6 años ENDES 2019. Recuperado de:

https://www.inei.gob.pe/media/MenuRecursivo/publicaciones digitales/Est/Lib1735/Libro.pdf

Kamza, A. (2019). Attachment to mothers and fathers during middle childhood: An evidence from Polish sample. BMC Psychology, 7. https://doi.org/10.1186/s40359019-0361-5

MIDIS (2019a). Lineamientos para la Gestión Articulada Intersectorial e Intergubernamental Orientada a Promover el Desarrollo Infantil Temprano. Editorial Corporación Creagrama E.I.R.L Recuperado de: https://cdn.www.gob.pe/uploads/document/file/343252/LINEAMIENTOS-DIT.pdf

MIDIS (2019b). DS N 003-2019-MIDIS Estrategia de Gestión Territorial Primero la Infancia. Recuperado de: https://www.gob.pe/institucion/midis/normaslegales/285219-003-2019-midis

PCEI (2017). Programa Curricular de Educación Inicial-MINEDU. Recuperado de: http://www.minedu.gob.pe/curriculo/pdf/programa-curricular-educacion-inicial.pdf

Pérez-Escamilla, R., Rizzoli-Córdoba, A., Alonso-Cuevas, A., \& Reyes-Morales, H. (2017). Avances en el desarrollo infantil temprano: desde neuronas hasta programas a gran escala. Boletín Médico Del Hospital Infantil de México, 74(2), 86-97. https://doi.org/10.1016/i.bmhimx.2017.01.007 
Ring, E. \& O'Sullivan, L. (2018). Dewey: A panacea for the "schoolification" epidemic. International Journal of Primary, Elementary and Early Years Education, 46(4): 402-410. https://doi.org/10.1080/03004279.2018.1445474

Rodríguez Jiménez, A y Pérez Jacinto, AO (2017). Métodos científicos de indagación y de construcción del conocimiento. Revista Escuela de Administración de Negocios; 82:1-26. Universidad EAN Bogóta, Colombia. Recuperado de: https://www.redalyc.org/pdf/206/20652069006.pdf

Sánchez Flores, F A (2019). Fundamentos epistémicos de la investigación cualitativa y cuantitativa: consensos y disensos. Revista Digital de Investigación en Docencia Universitaria, 13(1), 102-122. https://dx.doi.org/10.19083/ridu.2019.644

SIREPI (2020). Sistema Regional de Primera Infancia. Piura. Recuperado de: https://www.regionpiura.gob.pe/desarrollo-social/sirepi

UNICEF. (2017). La violencia en la primera infancia. Recuperado de: https://www.unicef.org/lac/sites/unicef.org.lac/files/201803/20171023 UNICEF LACRO FrameworkViolencia ECD ESP.pdf

Vásquez, A. (2020). El Inventario de Desarrollo Infantil y la evaluación sistemática del desarrollo en contextos educativos. Teoría, creación e implementación. Universidad de la República, Uruguay. Recuperado de: https://psyarxiv.com/xg2hi/

Verch, K. (2017). Primeira Infância Melhor Transformando la atención a los primeros años de vida en América Latina: retos y conquistas de una política pública en el Sur de Brasil. [Monografía del Banco Interamericano de Desarrollo]. Recuperado de: https://publications.iadb.org/publications/spanish/document/Primeira-Inf\%C3\%A2nciaMelhor-Transformando-la-atenci\%C3\%B3n-a-los-primeros-a\%C3\%B1os-de-vida-enAm\%C3\%A9rica-Latina-Retos-y-conquistas-de-una-pol\%C3\%ADtica-p\%C3\%BAblica-en-el-surde-Brasil.pdf 